\title{
Hybrid memristor-CMOS implementation of logic gates design using LTSpice
}

\author{
Wan Mohd Hashimi Wan Mohamad Sharif, Mohd Faizul Md Idros, Syed Abdul Mutalib Al-Junid, \\ Fairul Nazmi Osman, Abdul Hadi Abdul Razak, Abdul Karimi Halim, Muhammad Adib Harun \\ Electronic Architecture and Application Research Group (EArA), Faculty of Electrical Engineering, \\ Universiti Teknologi MARA, Malaysia
}

\begin{tabular}{l} 
Article Info \\
\hline Article history: \\
Received Apr 14, 2020 \\
Revised Dec 15, 2020 \\
Accepted Dec 26, 2020 \\
\hline
\end{tabular}

Keywords:

CMOS

Half adder

Logic gate

LTSpice

Memristor

\begin{abstract}
In this paper, a hybrid memristor-CMOS implementation of logic gates simulated using LTSpice. Memristors' implementation in computer architecture designs explored in various design structures proposed by researchers from all around the world. However, all prior designs have some drawbacks in terms of applicability, scalability, and performance. In this research, logic gates design based on the hybrid memristor-CMOS structure presented. 2-inputs AND, OR, NAND, NOR, XOR, and XNOR are demonstrated with minimum components requirements. In addition, a 1-bit full adder circuit with high performance and low area consumption is also proposed. The proposed full adder only consists of 4 memristors and 7 CMOS transistors. Half design of the adder base on the memristor component created. Through analysis and simulations, the memristor implementation on designing logic gates using memristor-CMOS structure demonstrated using the generalized metastable switch memristor (MSS) model and LTSpice. In conclusion, the proposed approach improves speed and require less area.
\end{abstract}

This is an open access article under the CC BY-SA license.

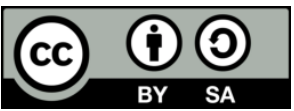

Corresponding Author:

Mohd Faizul bin Md Idros

Electronic Architecture and Application Research Group (EArA)

Faculty of Electrical Engineering

Universiti Teknologi MARA, Malaysia

Email: faizul_san287@uitm.edu.my

\section{INTRODUCTION}

Memristors have made a huge breakthrough in computer architecture designs because of its nonvolatility behavior. A lot of researches had reported their outcome even though is not commercially available [1-4]. Much evidence proved that using analog-based circuit operation will results in much faster data processing compared to current digital-based operation. It can remember its past value even when the power supply disconnected or turned off. Its resistance depends on the history of the applied inputs: Voltage or current. Moreover, it is predicted that memristors will be the key to designing analog-based data processing as shown in (1) and (2).

$$
\begin{aligned}
& V_{\text {out }, A N D}=\frac{R_{\text {on }}}{R_{\text {on }}+R_{\text {off }}} V_{\text {high }} \cong 0 \\
& V_{\text {out }, \text { OR }}=\frac{R_{\text {off }}}{R_{\text {off }}+R_{\text {on }}} V_{\text {high }} \cong V_{\text {high }}
\end{aligned}
$$


Therefore, hybrid CMOS circuits were proposed by many researchers for performance improvement [5-11]. The combination with memory characteristics of the memristors creates a unique opportunity for the next generation computers in which memory and logic devices blended to avoid a data bandwidth limitation between processing units and the memory $[12,13]$. Simulating the developed model of memristor was the best way to monitor the electrical properties as compared with the CMOS properties [14-18].

In this paper, a Hybrid CMOS-memristor for Half-Adder is implemented using LT-SPICE. The implementation started with the simulation of the AND and XOR logic gate. This to make sure that every hybrid component is working. Then, the comparison in terms of area and delay consumption for the proposed full adder are collected. Compared with previous works, this approach presents considerably less delay and area.

\section{METHODOLOGY}

The design phase can be divided into several stages. The initial stage focuses on designing the static CMOS logic gates such as AND, XOR, and Full Adder. The second stage is to implement the memristor equivalent circuits for the AND, XOR and Full Adder. The circuits are built as per circuit diagrams. All of the requirements and limitations are considered. Moreover, all of the gates are simulated in LT Spice. The output waveforms are analyzed and verified working as per expectation. All of the results are compared with previous researches.

\subsection{Implementation of memristor AND logic gate and XOR logic gate}

Figure 1 shows an AND gate using memristor the resistor after the input has been replaced with memristor to see how it work. As for R1 is using resistor because the memristor input cannot come from other memristor output, the output that will produced is unpredictable. The output status of a digital logic AND gate returns "LOW" only when any of its inputs are at a " 0 " level of logic. In other words, each LOW input will give a LOW output for a logic AND gate.

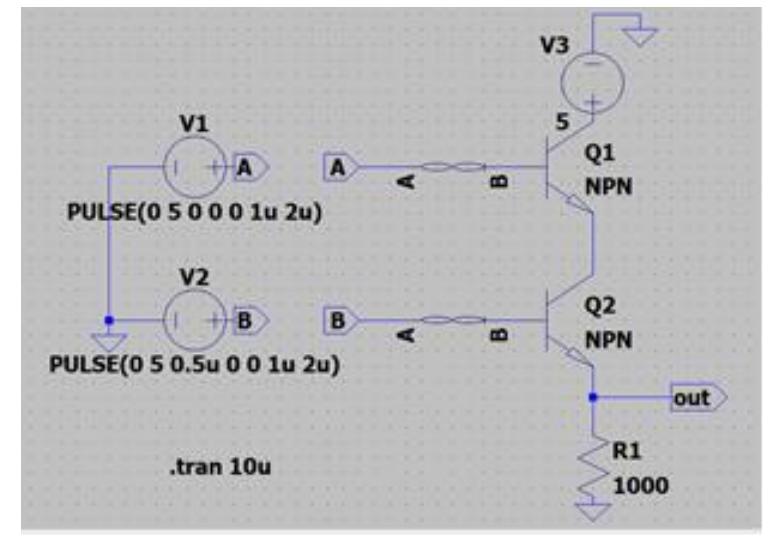

Figure 1. AND gate memristor

The logic AND Boolean expression for a digital logic AND gate is given as A.B $=\mathbf{Q}$. The operation of a digital 2-input logic AND gate can then be defined as: "If both A and B are valid, then Q is true". Exclusive-OR gate feature is accomplished by integrating regular logic gates to produce more complicated gate functions that are commonly used in the creation of arithmetic logic circuits, computational logic comparators, and error detection circuits. The "Exclusive-OR" two-input gate is a two-adder module, providing the sum of two binary numbers, rendering it more complicated in nature than other basic logic gate forms.

\subsection{Memristor full adder logic gate}

Using previous designs of AND, OR, and XOR, a hybrid memristor-CMOS full adder constructed using 4 memristors and 7 CMOS transistors. In this proposed full adder, the Sum bit circuit and Carry out bit separated from each other for silmulation purposes using LT-Spice as shown in Figure 2. An AND gate memristor used to produce a carry output, while the XOR gate using memristor to produce sum output. The full adder is used to add two single-digit binary numbers which contribute to a two-digit production. 

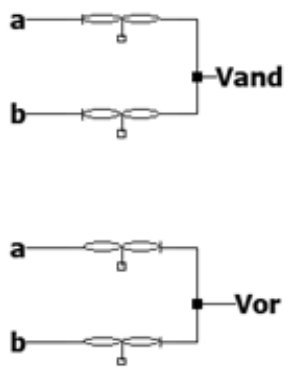

(a)

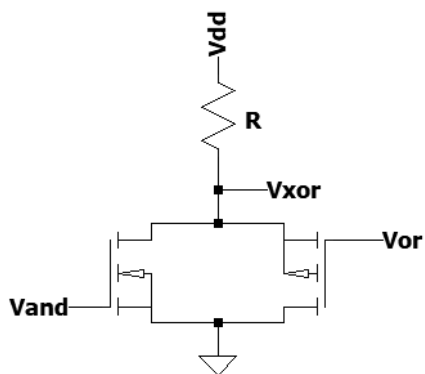

(c)

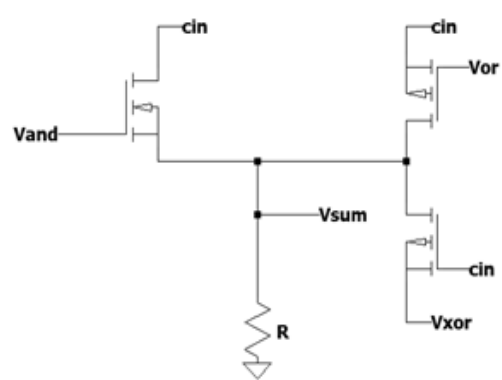

(b)

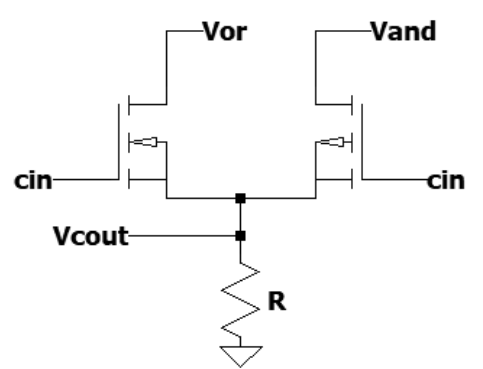

(d)

Figure 2. Full adder with separated element. (a) AND and OR circuit, (b) XOR circuit, (c) sum bit circuit, (d) Carry out bit circuit, $\mathrm{R}=10 \mathrm{k} \Omega, \mathrm{Vdd}=5 \mathrm{~V}$

\section{RESULTS AND DISCUSSION}

All of the simulations in this work are done using the generalized metastable switch memristor (MSS) model proposed by Knowm Inc. [19]. The MSS model is capable of modeling the memristor's hysteresis behavior precisely and is suitable for RRAM technology. This model was introduced by Knowm referring to the previously proposed model by Biolek [20] and VTEAM [21]. The parameters are set as follows; $R_{\text {on }}=500 \Omega, R_{\text {off }}=150 \mathrm{k} \Omega, V_{\text {on }}=2.5 \mathrm{~V}, V_{\text {off }}=2.5 \mathrm{~V}$. The current-voltage relationship of this memristor is shown in Figure 3. The software used for these simulations is LTSpice XVII, a free high-performance SPICE simulation software for Windows. The resistor used for all of the voltage reading purposes is $10 \mathrm{k} \Omega$. The simulation results for one MSS memristor model are shown in Figures 3 and 4. According to the hysteresis loop of this memristor model, it is visible that it follows the parameters set earlier with both $\mathrm{V}_{\text {on }}$ and $\mathrm{V}_{\text {off }}$ are equal to $2.5 \mathrm{~V}$.

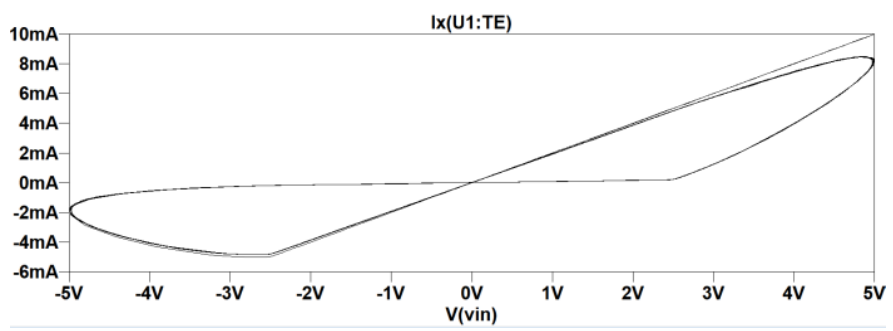

Figure 3. Hyteresis loop simulation

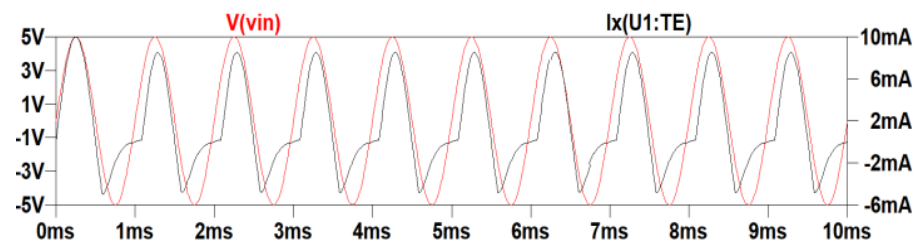

Figure 4. MSS model simulation 


\subsection{Memristor-CMOS XOR design}

A high-performance CMOS XOR logic gate design obtained using AND gate that is connected to a single NMOS and OR gate that is connected to a single PMOS. This design can be implemented using four memristors combined with two CMOS as a switch as shown in Figure 5. The basic operation of an XOR logic gate is to output zero voltage if the two applied inputs are equal. Figures 6 and 7 show the operation of this different input XOR. When the two inputs are both high, the AND circuit will output high and provide the voltage to the NMOS which operates on high voltage. When NMOS conduct, PMOS does not conduct and the output voltage is equal to zero. Correspondingly, when inputs applied are both low, the OR gate will output low and provide voltage to the PMOS which operates on low voltage. When the PMOS conduct, NMOS does not conduct and the output voltage is equal to zero. The simulation waveforms for the XOR circuit shown in Figure 8.
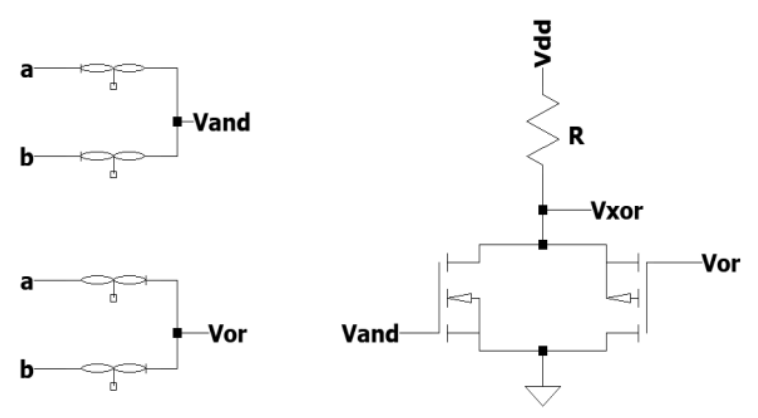

Figure 5. Schematic of memristor-CMOS XOR2 circuit, $\mathrm{R}=10 \mathrm{k} \Omega, \mathrm{Vdd}=5 \mathrm{~V}$

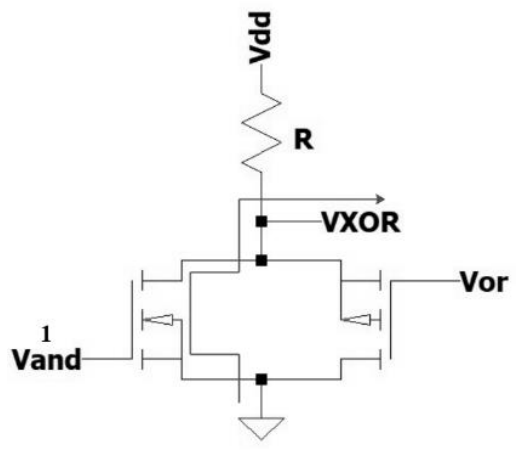

Figure 6. The state when both inputs are 1

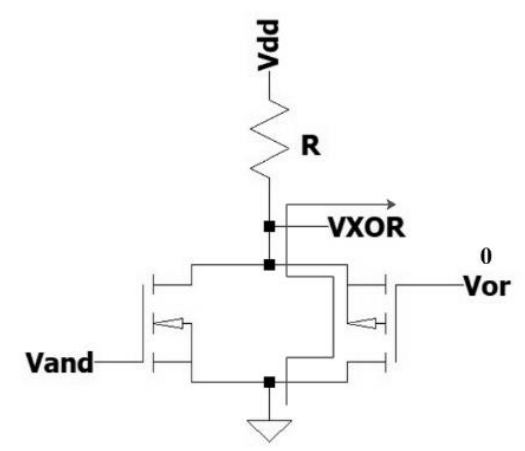

Figure 7. The state when both inputs are 0

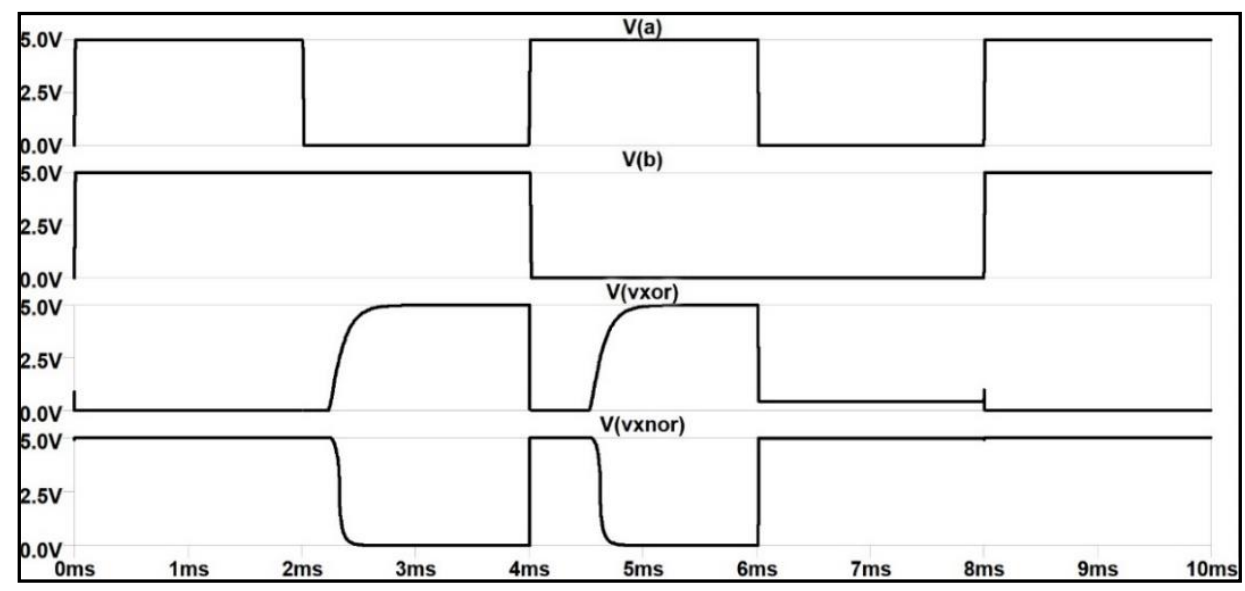

Figure 8. Simulation waveforms of XOR and XNOR logic gates shown in Figures 7 and 8 
The input voltages are represented by $\mathrm{V}(\mathrm{a})$ and $\mathrm{V}(\mathrm{b})$. The XNOR gate is achieved using additional CMOS inverter. The waveform is tabulated in Table 1 with logic 1 is $5 \mathrm{~V}$ and logic 0 is $0 \mathrm{~V}$. The presented XOR and XNOR have simple topology and fewer components that constructed with 4 memristors and 2 CMOS transistors, another additional 2 CMOS transistors are required as an inverter for XNOR circuit. This topology reduces CMOS usage and hence leads to lower area and power consumption

Table 1. Tabulated data of somulation waveforms in Figure 9

\begin{tabular}{ccccc}
\hline Time $(\mathrm{ms})$ & \multicolumn{2}{c}{ Input } & \multicolumn{2}{c}{ Output } \\
& $\mathrm{V}(\mathrm{a})$ & $\mathrm{V}(\mathrm{b})$ & $\mathrm{V}($ xor $)$ & $\mathrm{V}$ (xnor) \\
\hline $0-2$ & 1 & 1 & 0 & 1 \\
$2-4$ & 0 & 1 & 1 & 0 \\
$4-6$ & 1 & 0 & 1 & 0 \\
$6-8$ & 0 & 0 & 0 & 1 \\
\hline
\end{tabular}

\subsection{Memristor-CMOS full adder design}

The presented Sum bit circuit is made up of 3 CMOS transistors and output from the AND, OR and XOR circuit. The operation of the Sum bit circuit shown in Figure 9. The Sum bit is equal to high when the inputs (Cin.B.A) are equal to 001, 010, 100, and 111. Meanwhile, the presented Carry out circuit is consists of 2 CMOS transistors and output from the AND and OR circuit. The operation of this Carry out circuit explained in Figure 10 where Carry out bit is equal to high when the inputs (Cin.B.A) are equal to 011, 101, 110, and 111. The operation of this circuit shown in Figure 11. The operation of this full adder circuit shown in the simulation results shown in Figure 11.

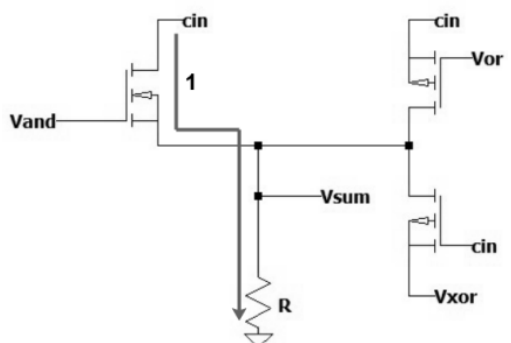

(a)

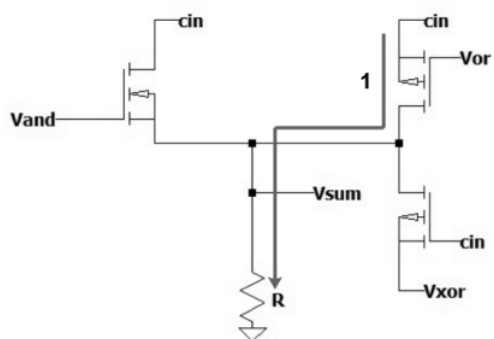

(b)

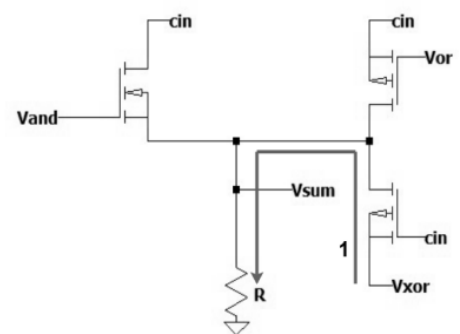

(c)

Figure 9. The path when the sum bit is high (1), (a) Cin.B.A (111), (b) Cin.B.A (100),

(c) Cin.B.A (001, 010), the current flows is shown by the line with $\mathrm{R}=10 \mathrm{k} \Omega$

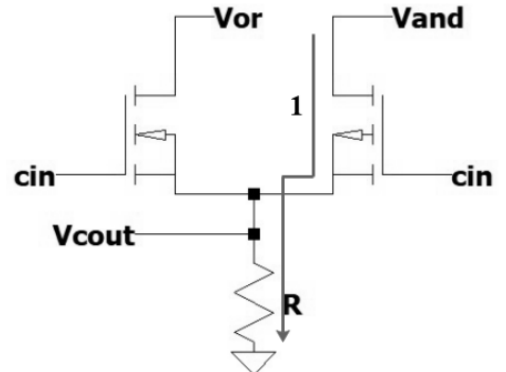

(a)

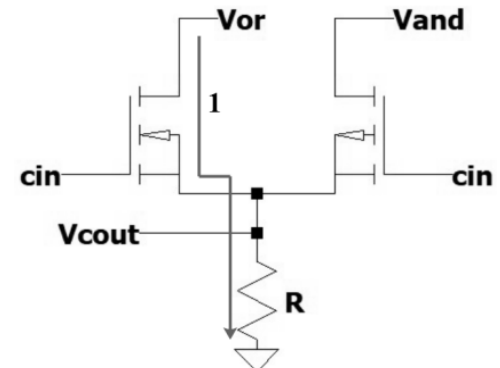

(b)

Figure 10. The path when the carry out bit is high (1), (a) Cin.B.A (011), (b) Cin.B.A (101, 110, 111), the current flows is as shown by the line with resistor $\mathrm{R}$ is equal to $10 \mathrm{k} \Omega$

Table 2 explains the tabulated data simulation waveforms of full adder from Figure 11. There are 8 different intervals to show 3-bit input from ' 000 ' to ' 111 '. The output V(sum) and V(cout) shows the output from the designed hybrid memristor-CMOS meets same as the normal Full Adder. 


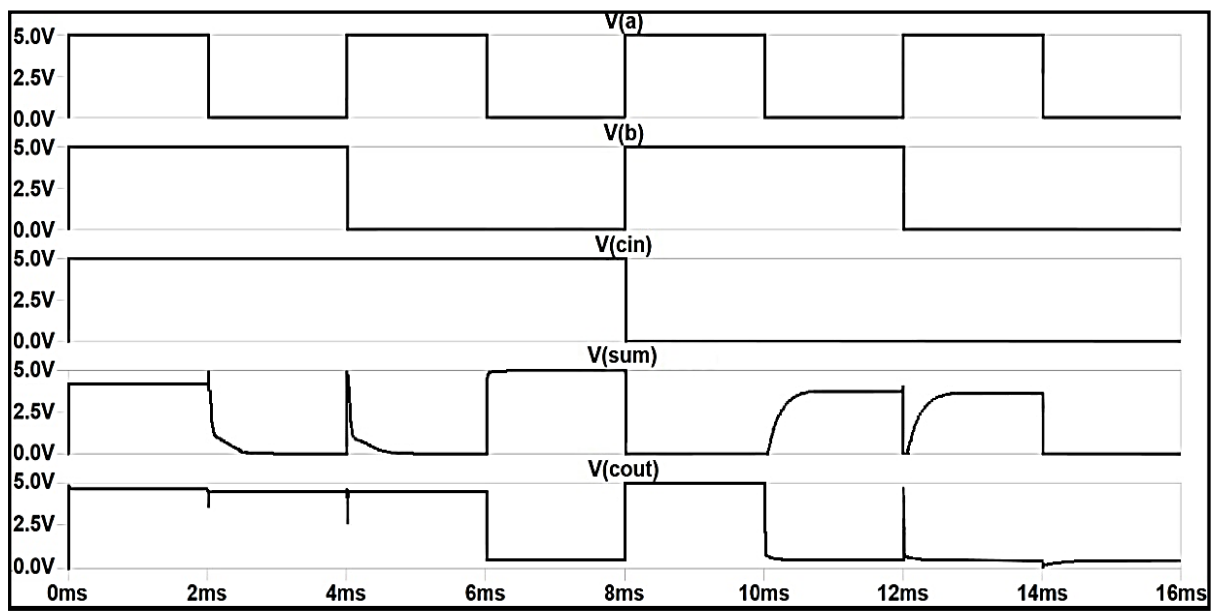

Figure 11. The simulation results of the full adder circuit in Figure 12, the input voltages are represented by $\mathrm{V}(\mathrm{a}), \mathrm{V}(\mathrm{b})$ and $\mathrm{V}(\mathrm{cin})$, in the simulation, logic 1 is $5 \mathrm{~V}$ and logic 0 is $0 \mathrm{~V}$

Table 2. Tabulated data of simulation waveforms in Figure 11

\begin{tabular}{cccccc}
\hline Time $(\mathrm{ms})$ & \multicolumn{3}{c}{ Input } & \multicolumn{2}{c}{ Output } \\
& $\mathrm{V}(\mathrm{a})$ & $\mathrm{V}(\mathrm{b})$ & $\mathrm{V}(\mathrm{cin})$ & $\mathrm{V}($ sum $)$ & $\mathrm{V}($ cout $)$ \\
\hline $0-2$ & 1 & 1 & 1 & 1 & 1 \\
$2-4$ & 0 & 1 & 1 & 0 & 1 \\
$4-6$ & 1 & 0 & 1 & 0 & 1 \\
$6-8$ & 0 & 0 & 1 & 1 & 0 \\
$8-10$ & 1 & 1 & 0 & 0 & 1 \\
$10-12$ & 0 & 1 & 0 & 1 & 0 \\
$12-14$ & 1 & 0 & 0 & 1 & 0 \\
$14-16$ & 0 & 0 & 0 & 0 & 0 \\
\hline
\end{tabular}

By combining the circuit of Sum bit and Carry out bit together, the complete full adder circuit is made up of 7 CMOS transistors and 4 memristors. This proposed full adder circuit has fewer components compared with other structures and with fewer components, it leads to less delay, less power, and less area consumption and hence, a high-performance full adder circuit was proposed.

\subsection{Comparison and discussion}

A summary and comparison in terms of area and delay consumption for the proposed full adder are shown in Table 3. The comparison is done with other memristors based design structures for basic logics and Full Adders. In terms of delay, this proposed full adder structure has a lower delay compared to MAGIC, IMPLY, and CRS with 4N delay. However, it is slower than the MRL and MAD structure. Meanwhile, in terms of area, this proposed structure has the smallest area consumption with only 14 components in total. With a smaller area, the power consumed by this structure is bounded to be low and efficient.

Table 3. Area and delay comparison of different Full Adders

\begin{tabular}{ccccc}
\hline & Delay & CMOS & Memristor & Resistor \\
\hline Proposed & 4 & 7 & 4 & 3 \\
CRS [22] & 14 & 0 & 16 & 1 \\
MPLY [23] & 19 & 0 & 28 & 5 \\
Improved IMPLY [24] & 10 & 0 & 9 & 1 \\
MAGIC [25] & 16 & 0 & 28 & 0 \\
MRL [26] & 1 & 8 & 18 & 0 \\
MAD [27] & 2 & 14 & 8 & 9 \\
\hline
\end{tabular}

\section{CONCLUSION}

Memristor has recently risen in popularity for its capability and potential to revamp the development of computer architecture in terms of power, area, and efficiency. However, more researches are still needed to fully utilize this missing circuit fundamental in terms of its possibility in replacing or accommodate current CMOS technology in both logic and memory applications. In this work, logic gates design based on the 
hybrid memristor-CMOS structure is demonstrated and analyzed. 2-inputs AND and OR gate is presented using 2 memristors and additional 2 CMOS transistors are required for NAND and NOR gate operation. Not only are that, a simple XOR logic made up of 4 memristors and 2 CMOS transistors also proposed. In addition, a full adder circuit was also proposed with a low delay and smallest area consumption that constructed with a total of 4 memristors and 7 CMOS transistors. In conclusion, logic gates design using hybrid memristor-CMOS structure has been successfully demonstrated and analyzed.

\section{ACKNOWLEDGEMENTS}

This project was supported by Ministry of Higher Education Malaysia under Fundamental Research Grant Scheme (FRGS/1/2019/TK04/UITM/02/28).

\section{REFERENCES}

[1] J. Jin and L. Cui, "Fully Integrated Memristor and Its Application on the Scroll-Controllable Hyperchaotic System," Complexity, vol. 2019, p. 4106398, 2019, doi: 10.1155/2019/4106398.

[2] R. S. Williams, "How we found memristor," IEEE SPECTRUM, pp. 29-35, Dec. 2008.

[3] Ş. Yener and H. Kuntman, "A New CMOS Based Memristor Implementation," International Conference on Applied Electronics, 2012.

[4] M. O.; V. G. ; S. K. ; S. K. ; J. M. ; E. M. ; A. Jabir, "The Missing Applications Found: Robust Design Techniques and Novel Uses of Memristors," IEEE 25th International Symposium on On-Line Testing and Robust System Design (IOLTS 2019), pp. 159-164, 2019.

[5] M. Sampath, P. S. Mane, and C. K. Ramesha, "Hybrid CMOS-Memristor based FPGA Architecture," International Conference on VLSI Systems, Architecture, Technology and Applications (VLSI-SATA) International, 2015.

[6] M. A. Abubaker Sasi, Amirali Amirsoleimani, Arash Ahmadi, "Hybrid Memristor-CMOS Based Linear Feedback Shift Register Design," 24th IEEE International Conference on Electronics, Circuits and Systems (ICECS), 2017.

[7] K. Smagulova, A. Tankimanova, and A. Pappachen James, "CMOS-Memristor Hybrid Integrated Pixel Sensors," IEEE International Symposium on Nanoelectronic and Information Systems CMOS-Memristor, pp. 34-37, 2016, doi: 10.1109/iNIS.2016.46.

[8] M. A. Mehri Teimoori, Arash Ahmadi, Shahpour Alirezaee, "A Novel Hybrid CMOS-Memristor Logic Circuit Using Memristor Ratioed Logic," IEEE Canadian Conference on Electrical and Computer Engineering (CCECE) 2016 IEEE Canadian Conference on Electrical and Computer Engineering (CCECE), 2016.

[9] A. H. A. Razak, et al., "Design of $5.8 \mathrm{GHz}$ Integrated Antenna on 180nm Complementary Metal Oxide Semiconductor (CMOS) Technology," IOP Conf. Ser. Mater. Sci. Eng., vol. 341, no. 1, 2018, doi: 10.1088/1757899X/341/1/012015.

[10] S. Mandal, I. University of Calcutta, Centre for Research in Nanoscience and Nanotechnology, Kolkata, and Jayee Sinha, Amlan Chakraborty, "Design of Memristor-CMOS based logic gates and logic circuits," Design of Memristor-CMOS based logic gates and logic circuits, 2019.

[11] C. L. and Q. X. S. Pi, P. Lin, H. Jiang, "Device engineering and CMOS integration of nanoscale memristors," IEEE International Symposium on Circuits and Systems (ISCAS), pp. 425-427, 2014, doi: 10.1109/ISCAS.2014.6865156.

[12] V. D. Tran and D. J. Park, "A survey of data recovery on flash memory," International Journal of Electrical and Computer Engineering, vol. 10, no. 1, pp. 360-376, 2020, doi: 10.11591/ijece.v10i1.pp360-376.

[13] N. Dib and U. Al-Sammarraie, "Optimal design of symmetric switching CMOS inverter using symbiotic organisms search algorithm," International Journal of Electrical and Computer Engineering,, vol. 10, no. 1, pp. 171-179, 2020, doi: 10.11591/ijece.v10i1.pp171-179.

[14] L. B. Gaurav Sharma, "CMOS-Memristor Inverter Circuit Design and Analysis Using Cadence Virtuoso," IEEE International Conference on Recent Advances and Innovations in Engineering (ICRAIE-2016), 2016.

[15] Thangkhiew, P. L., et al., "Efficient Implementation of Adder Circuits in Memristive Crossbar Array," IEEE Region 10 Conference (TENCON), pp. 207-212, 2017,

[16] K.-S. Min, Khoa Van Pham, "Non-Ideal Effects of Memristor-CMOS Hybrid Circuits for Realizing Multiple-Layer Neural Networks," IEEE International Symposium on Circuits and Systems (ISCAS) : proceedings : ISCAS, 2019.

[17] S Shin, K Kim, SM Kang, "Memristor-Based Fine Resolution Programmable Resistance and Its Applications," International Conference on Communications, Circuits and Systems, pp. 948-951, 2009.

[18] I. Vourkas and G. C. Sirakoulis, "Emerging memristor-based logic circuit design approaches: A review," IEEE Circuits and Systems Magazine, vol. 16, no. 3, pp. 15-30, 2016, doi: 10.1109/MCAS.2016.2583673.

[19] T. W. Molter and M. A. Nugent, "The generalized metastable switch memristor model," CNNA 2016; 15th International Workshop on Cellular Nanoscale Networks and their Applications, vol. 2016, pp. 65-66, 2016.

[20] D. Biolek, M. Di Ventra, and Y. V. Pershin, "Reliable SPICE simulations of memristors, memcapacitors and meminductors," Radioengineering, vol. 22, no. 4, pp. 945-968, 2013.

[21] S. Kvatinsky, M. Ramadan, E. G. Friedman, and A. Kolodny, "VTEAM: A General Model for Voltage-Controlled Memristors," IEEE Trans. Circuits Syst. II Express Briefs, vol. 62, no. 8, pp. 786-790, 2015, doi: 10.1109/TCSII.2015.2433536.

[22] Y. Yang, et al., "Complementary resistive switch-based arithmetic logic implementations using material implication," IEEE Trans. Nanotechnol., vol. 15, no. 1, pp. 94-108, 2016, doi: 10.1109/TNANO.2015.2504841. 
[23] A. Kolodny, et al., "Memristor-based material implication (IMPLY) Logic: Design Principles and Methodologies," IEEE Trans. Very Large Scale Integr. Syst., vol. 22, no. 10, pp. 2054-2066, 2013, doi: 10.1109/tvlsi.2013.2282132.

[24] P. Huang et al., "Reconfigurable Nonvolatile Logic Operations in Resistance Switching Crossbar Array for LargeScale Circuits," Adv. Mater., vol. 28, no. 44, pp. 9758-9764, 2016, doi: 10.1002/adma.201602418.

[25] S. Kvatinsky et al., "MAGIC-Memristor Aided LoGIC," IEEE Trans. Circuits Syst. II Express Briefs, vol. 61, no. 11, pp. 895-899, 2014.

[26] S. Kvatinsky, N. Wald, G. Satat, and E. G. Friedman, "MRL-Memristor Ratioed Logic Memristors are Useful for Logic," 13th International Workshop on Cellular Nanoscale Networks and their Applications, pp. 1-6, 2012.

[27] L. Guckert and E. E. Swartzlander, "MAD gates-Memristor logic design using driver circuitry," IEEE Trans. Circuits Syst. II Express Briefs, vol. 64, no. 2, pp. 171-175, 2017, doi: 10.1109/TCSII.2016.2551554.

\section{BIOGRAPHIES OF AUTHORS}
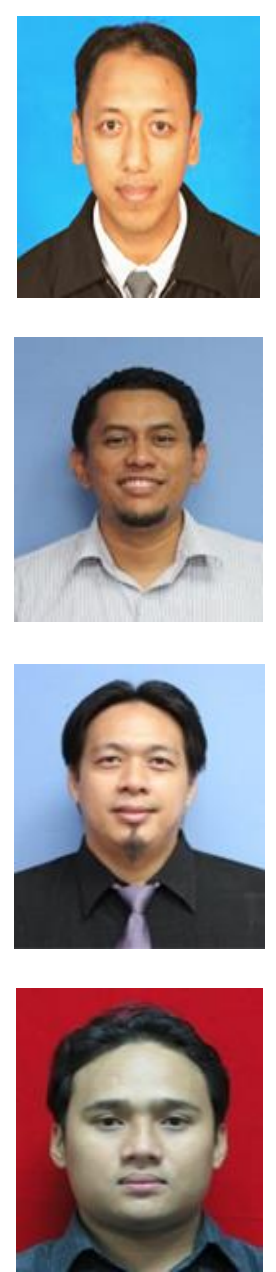

Mohd Faizul Md Idros was born in Banting, Selangor, Malaysia. He received the B.Engineeing from Universiti Teknologi MARA in 2002. Then he pursued his study and received his M. Sc. Degree and PhD from Universiti Kebangsaan Malaysia in 2006 and 2015 respectively. His research interests are in the field of Digital IC Design, Embedded System and Consumer Electronics.

Syed Abdul Mutalib Al-Junid recieved B.Eng from Universiti Teknologi MARA in 2006 and Master Engineering in 2010. Then he persue his study and obtained PhD in 2017. His research interests are in the field of IC Design, Embedded System Design, Microelectronic, IC Design, Engineering System Design, and Software Engineering.

Abdul Hadi Abdul Razak received the BEng (Hons) in Electrical Engineering from Universiti Teknologi MARA, Malaysia and Master in Microelectronics Engineering from Newcastle University, United Kingdom. He received his $\mathrm{PhD}$ in Electrical Engineering from Victoria University, Australia. Currently, he serves as an associate professor at Universiti Teknologi MARA, Malaysia. His current research is in the field of IC Design and Wireless Body Area Networks.

Adib Haron received the Bachelor of Engineering (Electrical) from Universiti Tun Hussein Onn Malaysia and Master of Engineering (Electrical - Electronics and Telecommunications) from Universiti Teknologi Malaysia. He serves as a senior lecturer at Universiti Teknologi MARA, Malaysia. His current research is in the field of memristor application. 